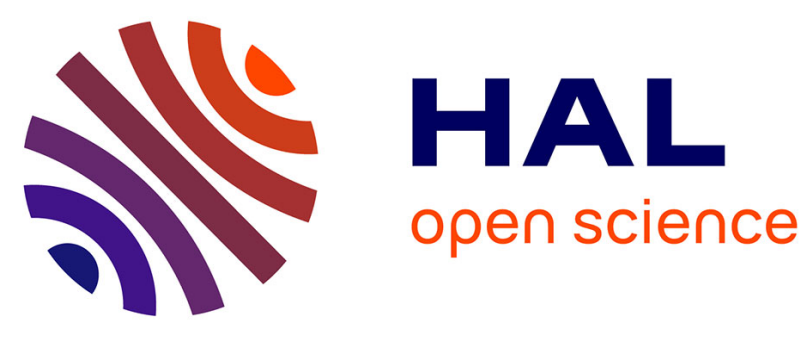

\title{
A transparent bilateral control scheme for a local teleoperation system using proportional-delayed controllers
}

José-Enrique Hernández-Díez, Silviu-Iulian Niculescu, César Fernando Méndez Barrios, Emilio-Jorge González-Galván, Raul Ignacio Hernández-Molinar

\section{- To cite this version:}

José-Enrique Hernández-Díez, Silviu-Iulian Niculescu, César Fernando Méndez Barrios, Emilio-Jorge González-Galván, Raul Ignacio Hernández-Molinar. A transparent bilateral control scheme for a local teleoperation system using proportional-delayed controllers. 12th IEEE International Conference on Control and Automation, ICCA 2016, Jun 2016, Katmandou, Nepal. pp.473-478, 10.1109/ICCA.2016.7505322 . hal-02274353

\section{HAL Id: hal-02274353 \\ https://hal-centralesupelec.archives-ouvertes.fr/hal-02274353}

Submitted on 17 Mar 2021

HAL is a multi-disciplinary open access archive for the deposit and dissemination of scientific research documents, whether they are published or not. The documents may come from teaching and research institutions in France or abroad, or from public or private research centers.
L'archive ouverte pluridisciplinaire HAL, est destinée au dépôt et à la diffusion de documents scientifiques de niveau recherche, publiés ou non, émanant des établissements d'enseignement et de recherche français ou étrangers, des laboratoires publics ou privés. 


\title{
A Transparent Bilateral Control Scheme for a Local Teleoperation System Using Proportional-Delayed Controllers
}

\author{
José Enrique Hernández-Díez ${ }^{1}$ Silviu-Iulian Niculescu ${ }^{2}$ César-Fernando Méndez-Barrios ${ }^{1}$ \\ Emilio Jorge González-Galván ${ }^{1}$ and Raúl Hernández-Molinar ${ }^{1}$
}

\begin{abstract}
This paper addresses the closed-loop stability analysis problem of a bilateral local teleoperation system in the presence of delays, with the purpose of maintaining a kinematic correspondence between a master and a slave device. The stability analysis is proposed as a general analysis in the controller's parameter-space under the assumption of two fixed delays, considering one due to the signal and communication processing and one defined as a design delay for the controller. Furthermore, a method for measuring the fragility of the controllers is also proposed. Finally, experimental results obtained from an experimental platform consisting of two Phantom Omni haptic devices and the Matlab-Simulink toolkit Phansim illustrate the performance of the proposed approach and the video of the experiments can be downloaded from the authors' dedicated website ${ }^{1}$.
\end{abstract}

\section{INTRODUCTION}

The development of teleoperation systems have become a very popular tool in multiple applications due to two main benefits: safety and precision. In other words, the safety of a human operator working in hostile environments can be greatly enhanced by using a teleoperation system [1], where the most important goal is to replace the direct human manipulation (see, [2], for further details), and perform in high precision operations [3] (and the references therein). This work pertains to the development of local teleoperation systems in which the main goals are to have a high precision system able to work with motion and force scaling. More precisely, in this paper we first focus in the ideal case in which the system must have a perfect degree of telepresence on the remote environment and a full sense feedback to the operator [4], [5]. As mentioned in [6], [3] a system having such a performance is nothing but a perfectly transparent system. Therefore, in order to create an ideal, efficient and confortable workspace, a teleoperation system must be perfectly transparent. There are two conditions required to achieve such a property: impedance matching and kinematic correspondence and they will be explicitly addressed in the sequel.

Some of the most widely used linear controllers in robotics are the so called P-D (Proportional-Derivative) controllers. Even though several techniques have been developed for a better performance, there are still situations in which the

\footnotetext{
${ }^{1}$ School of Engineering, Universidad Autónoma de San Luis Potosí, 8 Dr. Manuel Nava, 78290 San Luis Potosí, Mexico.

${ }^{2}$ Laboratoire des Signaux et Systèmes L2S (UMR CNRS 8506), CNRSCentraleSupelec-Université Paris-Sud, 3 rue Joliot Curie, 91192 Gif-surYvette, France.

${ }^{1}$ http://ciep.ing.uaslp.mx/electrica/RoboticsLab.php
}

noise produced by non-desired high frequency signals may lead to unexpected behaviors in some experimental tests. As suggested by Niculescu and Michiels [7] (see also [8]), in the context of the stabilization of chains of integrators, we may replace the derivative part of the controller by a delay block, and thus the PD controller may be replaced by a $P-\delta$ (Proportional-Delayed) controller. This paper explores such an idea in the context of a transparent bilateral control scheme including delays. In order to achieve a kinematic correspondence between the master and the slave in the delay case Liacu et al. [9] proposed a particular control scheme where the controllers for both master and slave are identical and of PD type. In this paper, we focus on the use of $P-\delta$ controllers instead of PD controllers and we are characterizing the stability regions in the controller parameter-space.

The main contribution of the paper is the stability analysis in the controller's parameter-space. We propose a straightforward method to compute the stability analysis by using $\mathscr{D}$-Partition curves [10], the Boundary Crossing Theorem [11] and the Implicit Function Theorem [12]. The main goal consists in finding all the controller's regions in the controller's parameter-space such that the closed-loop system is asymptotically stable. The proposed approach requires three steps:

1) Constructing the stability crossing boundaries in the appropriate parameter-space;

2) Computing the crossing direction in which, by using an appropriate choice of parameters, the roots move from one region to another by crossing the same boundary;

3) The explicit localization of the stability regions in the parameter-space.

In the procedure above, the first step defines a partition of the parameter-space defined by the controller's parameters in several regions, each region having a constant number of unstable roots for all the controller's gains inside the boundaries of the region. Such a method is nothing else than the $\mathscr{D}$-Decomposition method introduced by Neimark in 1949 ([10]) and largely discussed in the literature (see, for instance, [13]). Throughout this paper, a stability crossing boundary (curve) is interpreted as the set of parameters for which the characteristic equation of the closed-loop system has at least one root on the imaginary axis. Next, in step two, an argument based on the implicit function theorem is used to detect if a region contains more or less unstable 
roots compared to its neighboring regions. Finally, the third step allows detecting the regions in which the controllers guarantee the stability of the system in closed-loop.

Furthermore, in order to quantify the maximum allowable parameter deviation in the controller's gains of the system to ensure closed-loop stability, a geometrical method for measuring fragility is proposed. This method consists in computing the minimum distance from a point in the controller's parameter-space to the stability crossing boundaries (curves).

The experimental results presented in this work were obtained by using two Phantom Omni haptic devices, as the one depicted in Fig. 6, together with a test bench developed on the Phansim toolkit (for further details, the reader is referred to [14]) for Matlab-Simulink. The videos of some of the experiments performed in this frame can be founded at the authors dedicated website.

\section{Preliminary Results}

It is well known that the dynamics of a haptic device as the one shown in Fig. 6 can be modeled by considering a Lagrangian formulation [3], as follows:

$$
M(\theta) \ddot{\theta}+C(\theta, \dot{\theta}) \dot{\theta}+B=F_{\lambda},
$$

where $M$ is the inertia matrix, $C$ is the Coriolis matrix, $B$ is a vector associated to the effect of gravity, $F_{\lambda}$ is the torque input vector and $\theta$ is the angular position vector. The derived model is clearly non-linear. Now, inspired by the contributions of [3], [9], a few assumptions can be taken into account in order to describe the dynamics of the system as a decoupled time-invariant linear model. This is formed by the three mechanical admittances of each joint in the following form:

$$
P(s):=\frac{\Theta(s)}{\Lambda(s)}=\frac{1}{s(m s+b)},
$$

where each mechanical admittance $P(s)$ is described by the transfer function from each torque input $\Lambda(s)$ to its respectively angular position $\Theta(s)$ and depicts the behavior of each mechanical joint.

In our case, the main goal of the proposed control scheme is to achieve a kinematic correspondence between the master and slave device. As illustrated in Fig. 1, the main idea is to maintain a perfect bilateral position tracking under the interaction of the exogenous forces of the human and the remote environment on the master and the slave device, respectively. The ideal result is to have a complete perception of the remote environment to the human operator and a complete telepresence of the human operator on the remote environment (see, for instance, [6], [15]).

The bilateral control scheme proposed in this paper is shown in Fig. 2, where $\tau_{p}$ is considered as the delay due to signal processing, $\Lambda_{h}$ and $\Lambda_{e}$ are the exogenous torques related to the human operator and the remote environment,

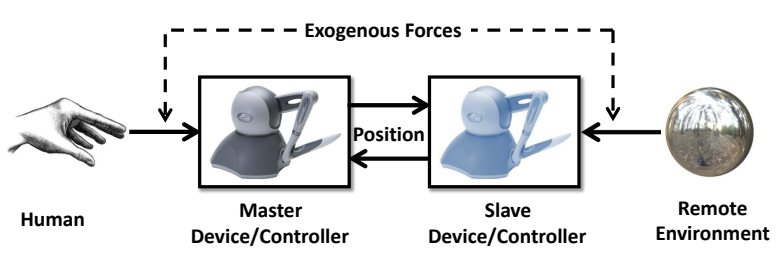

Fig. 1. Bilateral Control Scheme (Conceptual).

respectively, $P_{M}$ and $P_{S}$ are the mechanical admittances of the master and the slave device, respectively; furthermore, a similar notation is used for the controllers $C_{M}$ and $C_{S}$ and the angular positions $\Theta_{M}$ and $\Theta_{S}$. This scheme is a variation of the one presented in [9] for haptic-virtual systems, however here, we have considered the time delays due to signal processing and, instead of using a P-D controller, a $\mathrm{P}-\delta$ controller is proposed and discussed in detail later.

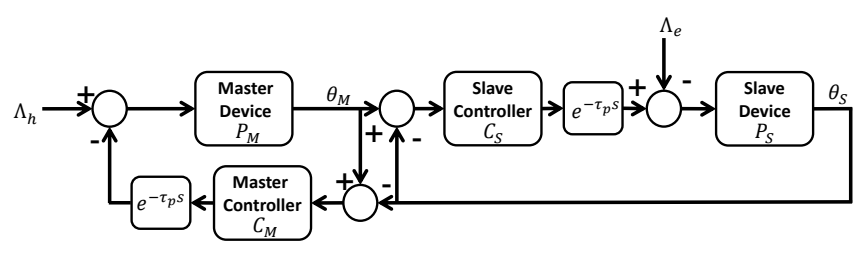

Fig. 2. Bilateral Control Scheme.

From Fig. 2, the equations describing the system response can be written as follows:

$\left[\begin{array}{cc}1+P_{M}(s) C_{M}(s) e^{-\tau_{p} s} & -P_{M}(s) C_{M}(s) e^{-\tau_{p} s} \\ -P_{S}(s) C_{S}(s) e^{-\tau_{p} s} & 1+P_{S}(s) C_{S}(s) e^{-\tau_{p} s}\end{array}\right]\left[\begin{array}{c}\Theta_{M}(s) \\ \Theta_{S}(s)\end{array}\right]=\left[\begin{array}{c}P_{M}(s) \Lambda_{h} \\ -P_{S}(s) \Lambda_{e}\end{array}\right]$.

In this paper, we consider the case in which $P(s):=$ $P_{M}(s)=P_{S}(s)$ and we fully characterize the system by setting $C(s):=C_{M}(s)=C_{S}(s)$. Then, taking into account the above considerations, the characteristic equation of the closed-loop system can be rewritten as follows:

$$
2 P(s) C(s) e^{-\tau_{p} s}+1=0 .
$$

\section{Main Results - Stability Analysis}

As mentioned in the Introduction, one of the main goals in this paper is to find a solution for the kinematic correspondence problem presented above by using the $\mathrm{P}-\delta$ (Proportional-Delayed) controller of the form:

$$
C(s)=K_{p}+K_{\delta} e^{-\tau s},
$$

where $K_{p}$ and $K_{\delta}$ are scalar gains and $\tau$ is a fixed and known parameter. In the sequel, without any loss of generality, we can say that the analysis presented in this paper can be used in any of the decoupled time-invariant systems of each joint (2). The characteristic function $\Delta: \mathbb{C} \mapsto \mathbb{C}$, of the system (3) can be rewritten as:

$$
\Delta(s):=m s^{2}+b s+2 e^{-\tau_{p} s}\left(K_{p}+K_{\delta} e^{-\tau s}\right),
$$


which has the structure of a quasi-polynomial, where $m$ and $b$ are parameters covering all the cases defined by (2).

\section{A. $\mathscr{D}$-partition Curves}

It is well known that a continuous variation of the parameters of a quasi-polynomial in which there is no change of degree implies a continuous variation of the roots of the quasi-polynomial in the complex plane (see, for instance [16], [13]). Let $\mathscr{T}$ denote the set of all $K=\left[K_{p}, K_{\delta}\right]^{T} \in \mathbb{R}^{2}$ forming the $K_{p}-K_{\delta}$ parameter-space such that (5) has at least one root on the imaginary axis. Also let $\Omega$ denote the frequency set of all real numbers $\omega$ also called crossing frequency set. Any $K \in \mathscr{T}$ is known as a crossing point and each subset of $\mathscr{T}$, which is continuous in $\mathbb{R}^{2}$, is known as a $\mathscr{D}$-partition curve or a stability crossing curve.

Remark 1: If $\omega$ is a real number, $K \in \mathbb{R}^{2}$ and $\tau$ is a fixed value such that $\tau \in \mathbb{R}_{+}$then $\Delta(-i \omega)=\overline{\Delta(i \omega)}$. Therefore, in order to find all the stability crossing curves, we only need to consider positive $\omega$.

Bearing in mind the discussion above, the following result describes the set of all crossing points:

Proposition 1: Let $\tau_{p}, \tau \in \mathbb{R}_{+}$and $\Omega:=\bigcup_{i} \Omega_{i}$, where

$$
\Omega_{i}:=\left\{\omega \in \mathbb{R}_{+} \cup\{0\} \mid \omega \in\left(\frac{\pi i}{\tau}, \frac{\pi(i+1)}{\tau}\right) ; \forall i \in \mathbb{Z}_{+} \cup\{0\}\right\} .
$$

Then, $\omega \in \Omega$ is a crossing frequency if and only if $K(\omega)=$ $\left[K_{p}(\omega), K_{\delta}(\omega)\right]^{T}$ given by:

$K_{p}(\omega)=\frac{m \omega^{2}\left(\cos \left(\tau_{p} \omega\right)-\cos \left(\left(2 \tau+\tau_{p}\right) \omega\right)\right)+b \omega\left(\sin \left(\tau_{p} \omega\right)-\sin \left(\left(2 \tau+\tau_{p}\right) \omega\right)\right)}{4 \sin ^{2}(\tau \omega)},(7)$

$K_{\delta}(\omega)=\frac{-m \omega^{2} \sin \left(\tau_{p} \omega\right)+b \omega \cos \left(\tau_{p} \omega\right)}{2 \sin \tau \omega}$,

defines a crossing point $K(\omega) \in \mathscr{T}$.

Proof: Consider the characteristic function (5), then, it is clear that all the crossing points $K \in \mathscr{T}$ are given by the pairs $K \in \mathbb{R}^{2}$ solving (5) for $s=i \omega$. It is easy to see that such solutions can be obtained by the solution of the following system of equations:

$$
\mathfrak{R}(\Delta(i \omega))=0, \quad \mathfrak{I}(\Delta(i \omega))=0 .
$$

This system of equations is solved for $K_{p}$ and $K_{\delta}$ straightforwardly by using simple algebraic manipulations. The solution of the system is presented in equations (7) and (8), respectively. Furthermore, from equations (7) and (8), it is easy to see that $K_{\omega}(\omega)$ is a real solution if and only if $\omega \neq n \frac{\pi}{\tau}$ for $n \in \mathbb{Z}^{+} \cup\{0\}$.

Remark 2: There exists some particular case in which a solution $K_{\omega}(\omega)$ is not well defined for $\omega=0$. In this case, the stability crossing curve related to $s=0$ can be found through (5) as:

$$
\Delta(0)=2 K_{p}+2 K_{\delta}=0,
$$

or the points on the line:

$$
K_{\delta}=-K_{p}
$$

Given all the crossing points $K$ and the crossing-frequency set $\Omega$, we can define each stability crossing curve through its continuity, as follows:

$$
\begin{gathered}
\mathscr{T}_{o}:=\left\{K \in \mathbb{R}^{2} \mid K_{\delta}=-K_{p}\right\}, \\
\mathscr{T}_{i}:=\left\{K_{\omega}(\omega) \in \mathbb{R}^{2} ; \forall \omega \in \Omega_{i}\right\},
\end{gathered}
$$

for $i \in \mathbb{Z}_{+} \cup\{0\}$. It is evident that

$$
\mathscr{T}=\bigcup_{i} \mathscr{T}_{i} \bigcup \mathscr{T}_{o}
$$

Given all the stability crossing curves in the $K_{p}-K_{\delta}$ parameter-space and considering the boundary crossing theorem (for further details, see, for instance [11]). It is clear that the $K_{p}-K_{\delta}$ parameter-space is partitioned by the stability crossing curves in stable and unstable regions and in which the characteristic equation (5) has a finite number of unstable roots.

\section{B. Crossing Directions}

Proposition 2: A simple root, or a pair of simple roots of function (5), moves from the left half complex plane (LHP) to the right half complex plane (RHP) as $K$ crosses a stability crossing curve with $\omega=0$ or $\omega \neq 0$, respectively, in the increasing direction of $K_{\chi}$ for $\chi \in\{p, \delta\}$ if:

$$
\begin{aligned}
\mathscr{C}_{\chi}= & m \omega S\left(\eta_{\chi}, \omega\right)-\frac{b}{2} C\left(\eta_{\chi}, \omega\right)+\tau_{p} K_{p} F\left(\tau, \omega, \eta_{\chi}\right) \\
& +\left(\tau_{p}+\tau\right) K_{\delta} F\left(\tau_{p}+\tau, \omega, \eta_{\chi}\right)>0,
\end{aligned}
$$

where:

$$
F\left(y, \omega, \eta_{\chi}\right)=\cos (y \omega) C\left(\eta_{\chi}, \omega\right)+\sin (y \omega) S\left(\eta_{\chi}, \omega\right)
$$

$$
C\left(\eta_{\chi}, \omega\right)=\cos \left(\left(\tau_{p}+\eta_{\chi} \tau\right) \omega\right), \quad S\left(\eta_{\chi}, \omega\right)=\sin \left(\left(\tau_{p}+\eta_{\chi} \tau\right) \omega\right)
$$

and where the indicative function $\eta_{\chi}$ is defined as:

$$
\eta_{\chi}=\left\{\begin{array}{lll}
0 & \text { if } & \chi=p \\
1 & \text { if } & \chi=\delta
\end{array}\right.
$$

Furthermore, the crossing is from the RHP to the LHP if the inequality (14) is reversed.

Proof: The proof follows straightforwardly from the fact that the derivative of the implicit function $s\left(K_{x}\right)$ along $K_{x}$ is given by

$$
\frac{d s}{d K_{x}}=-\frac{\frac{\partial \Delta}{\partial K_{x}}}{\frac{\partial \Delta}{\partial s}} .
$$

Thus, the real part of the previous derivative evaluated on a stability crossing point for $x=p$ and $x=\delta$ is computed as:

$$
\Re\left\{\frac{d s}{d K_{p}}\right\}, \quad \Re\left\{\frac{d s}{d K_{\delta}}\right\},
$$

respectively. The sign of both equations can be described by the inequality (14) which is arranged in a practical structure by the indicative function $\eta_{x}$ previously defined. 
In order to derive an algorithm to characterize the stability regions by a number of unstable roots (invariant in each region), let's assume that we have $\ell$-regions $\mathscr{R}_{1}, \mathscr{R}_{2}, \ldots, \mathscr{R}_{\ell}$, with $\ell \geq 2$. Without any loss of generality, assume that $\mathscr{R}_{1}$ and $\mathscr{R}_{2}$ are the first two neighboring regions (relabeled if necessary) of interest (for instance, closest to the origin), and let $K^{(j)}$ be a point on the boundary of regions $\mathscr{R}_{j}$ and $\mathscr{R}_{j+1}$. We have the following algorithm:

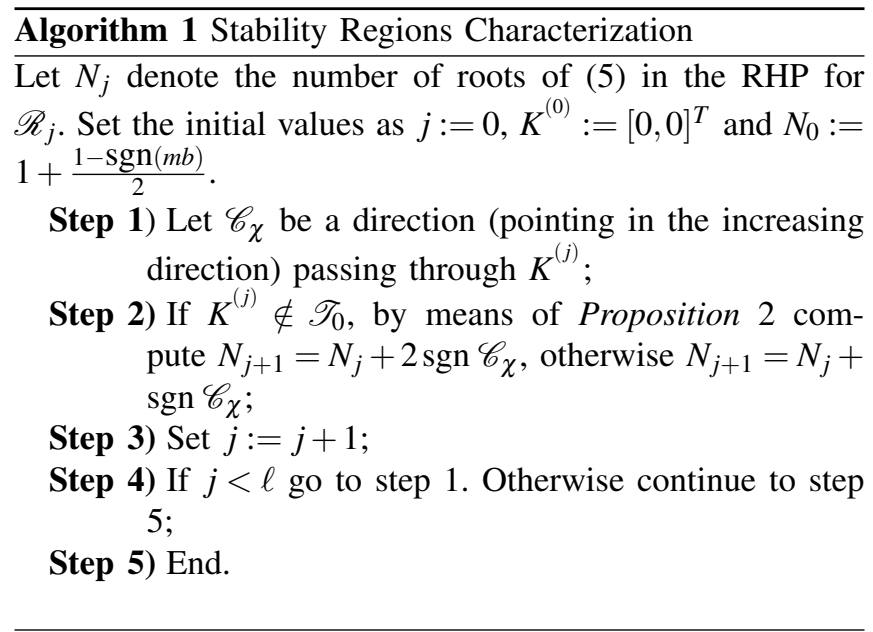

Remark 3: Observe that, for $\left[K_{p}, K_{\delta}\right]^{T}=K^{(0)},(5)$ is a second-degree polynomial having a root on the origin, and consequently the second root can be determined by simple inspection.

The above iterative process characterize directly the qualitative behavior of the system response as the controller gains traverse the $K_{p}-K_{\delta}$ parameter-space.

\section{Fragility}

Consider now the fragility problem, which is the problem of computing the maximum controller parameters' deviation without loosing the closed-loop stability given by some known values $K^{*}=\left(K_{p}^{*}, K_{\delta}^{*}\right)^{T}$, such that the roots of the equation:

$$
\Delta\left(s ; K_{p}^{*}, K_{\delta}^{*}\right)=0,
$$

are located in $\mathbb{C}_{-}$(that is the closed-loop system is asymptotically stable). This is analogous to find the maximum parameter deviation $d \in \mathbb{R}_{+}$such that the roots of (18) stay located in $\mathbb{C}_{-}$for all controllers $K$ satisfying:

$$
\sqrt{\left(K_{p}-K_{p}^{*}\right)^{2}+\left(K_{\delta}-K_{\delta}^{*}\right)^{2}}<d .
$$

First, let us introduce some notations: for a fixed $K^{*}:=$ $\left(K_{p}^{*}, K_{\delta}^{*}\right)^{T} \in \mathbb{R}^{2}$ and $K(\omega)=\left(K_{p}(\omega), K_{\delta}(\omega)\right)^{T}$ (taken directly from Proposition 1), introduce the function $\xi: \mathbb{R}_{+} \rightarrow \mathbb{R}_{+}$, as:

$$
\xi(\omega):=\sqrt{\left(K_{p}(\omega)-K_{p}^{*}\right)^{2}+\left(K_{\delta}(\omega)-K_{\delta}^{*}\right)^{2}} .
$$

We have the following:

Proposition 3: Let $K^{*}=\left(K_{p}^{*}, K_{\delta}^{*}\right)^{T}$ be a stabilizing controller then, the maximum parameter deviation : " $d$ " of $K^{*}$ without loosing the property of stability can be computed by:

$$
d=\min \left\{d_{\ell}, d_{o}\right\},
$$

where $d_{\ell}$ and $d_{o}$ are given by:

$$
d_{\ell}=\min _{\omega \in \Omega_{f}}\{\xi(\omega)\}, \quad d_{o}=\frac{1}{\sqrt{2}}\left(K_{p}^{*}+K_{\delta}^{*}\right),
$$

where $\Omega_{f}$ denote the set of all roots of $f(\omega)$ defined as:

$$
f(\omega):=\left\langle\left(K(\omega)-K^{*}\right), \frac{d K(\omega)}{d \omega}\right\rangle,
$$

and $\langle\cdot, \cdot\rangle$ refers to the inner product.

Proof: Taking into account the fact that $K^{*}$ belongs to a stability region delimited by the stability crossing curves, it is clear that the system will loose its stability if the controller $K^{*}$ has a parameter deviation such that it crosses for at least one of its boundaries. Therefore, the objective is to compute the minimal distances between $K^{*}$ and the different boundaries of the stability region. In order to compute the minimal distance between a point $K^{*}$ and the stability crossing curves with $\omega \neq 0$, we need to identify the points $K(\omega)$ in which the tangent vectors to the curve are orthogonal to $K(\omega)-K^{*}$ which are the roots of equation (23) and where the minimum distance $d_{\ell}$ to a stability crossing curve with $\omega \neq 0$ is given by (22).

On the other hand, notice that the borders of the stability crossing curve related to $\omega=0$ are described by equation (10). Now substituting in (20), it leads to:

$$
\xi(0)=\sqrt{2 K_{p}^{2}+2\left(K_{\delta}^{*}-K_{p}^{*}\right) K_{p}+\left(K_{\delta}^{*^{2}}+K_{p}^{*^{2}}\right)},
$$

which is the distance between the point $K^{*}$ and the stability crossing curve related to $\omega=0$, the point $K$ in which $\xi(0)$ is globally minimal is given by the solution of the following equation:

$$
\frac{d \xi^{2}(0)}{d K_{p}}=4 K_{p}+2\left(K_{\delta}^{*}-K_{p}^{*}\right)=0,
$$

then, this minimal value is $d_{o}$ and can be obtained by substituting the solution in equation (24) which is the right part of (22). Finally, the minimal distance $d$ can be computed by using (21).

\section{ILLUSTRATIVE AND EXPERIMENTAL RESULTS}

For our experimental setup the delay is estimated to be $\tau_{p}=0.001$ seconds. The parameters of the system are identified by using a methodology based on a least squares algorithm and are shown in table I. In all following examples, we propose a fixed delay value $\tau_{d}=0.1$ seconds in the design of the P- $\delta$ controller.

TABLE I

\begin{tabular}{ccc}
\multicolumn{3}{c}{ PARAMETERS OF THE SYSTEM. } \\
\hline Joint & $m$ & $b$ \\
\hline 1 & 0.0131 & 0.0941 \\
2 & 0.0307 & 0.1719 \\
3 & $4.28 \times 10^{-5}$ & 0.1066 \\
\hline
\end{tabular}




\section{A. Stability Analysis}

In order to describe the process of finding the stability regions, the system formed by the joint one is considered as an illustrative example. Figure 3 and table II shows the process of finding the stability regions as established in the Algorithm 1, where $N$ denotes the number of unstable roots in the RHP for any $K$ on each region or in the origin.

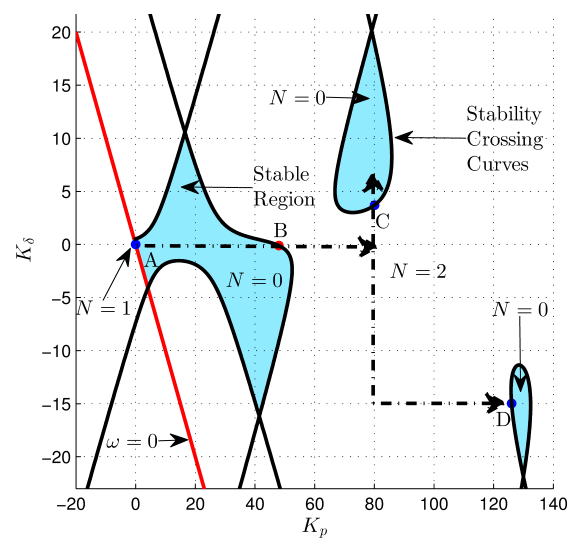

Fig. 3. Crossing Directions Analysis for the Joint One

TABLE II

Crossing Directions.

\begin{tabular}{ccccccc}
\hline Point & $K_{p}$ & $K_{\delta}$ & $\omega$ & $\eta_{\chi}$ & $\mathscr{C}_{\chi}$ & Sign \\
\hline$A$ & 0 & 0 & 0 & 0 & -0.0471 & - \\
$B$ & 47.9954 & -0.1070 & 85.5119 & 0 & 0.0282 & + \\
$C$ & 80.0735 & 3.7006 & 110.6978 & 1 & -0.9807 & - \\
$D$ & 126.0201 & -14.9816 & 131.9937 & 0 & -0.9285 & - \\
\hline
\end{tabular}

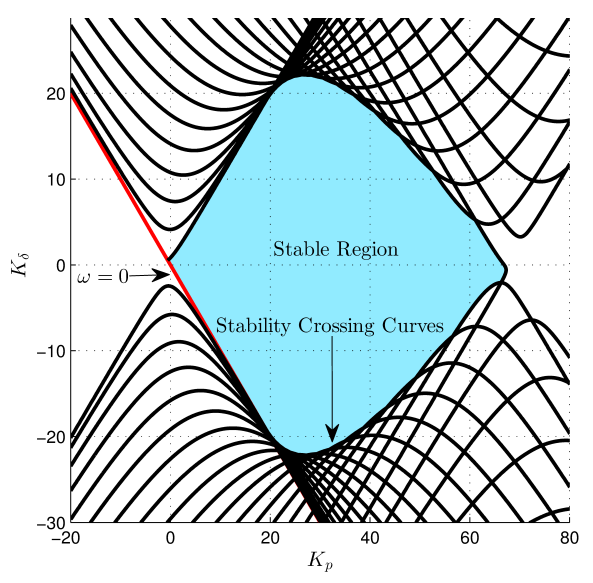

Fig. 4. Stability Regions for the Joint Three

\section{B. Fragility}

In this example, we measure the fragility of three different stabilizing controllers $K_{1}^{*}, K_{2}^{*}$ and $K_{3}^{*}$ for the joint two. The results are derived by using Proposition 3 and are shown in Tab. III. In order to illustrate this results, Fig. 5 shows three circles centered at $K_{1}^{*}, K_{2}^{*}$ and $K_{3}^{*}$ with radius $d$ for each different analysis.

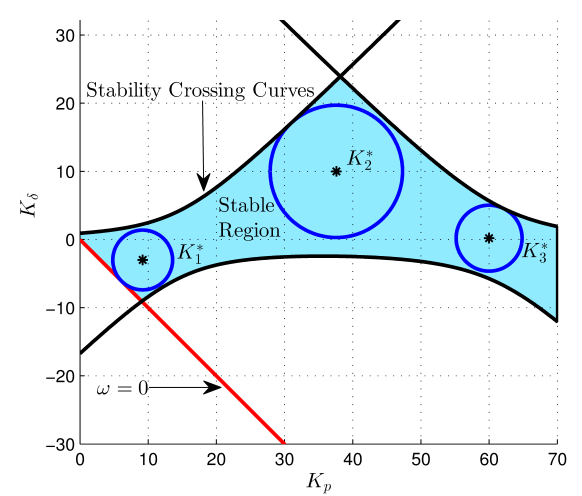

Fig. 5. Fragility Results for the Joint Two

TABLE III

\begin{tabular}{cccccccc}
\multicolumn{7}{c}{ FRAGILITY RESULTS. } \\
\hline$K$ & $K_{p}$ & $K_{\delta}$ & $\omega$ & $\xi(\omega)$ & $d_{\ell}$ & $d_{o}$ & $d$ \\
\hline$K_{1}^{*}$ & 9.2 & -3 & \pm 34.7359 & 27.5812 & 4.9366 & 4.3841 & 4.3841 \\
$K_{2}^{*}$ & 37.6 & 10 & \pm 30.15 & 8.2095 & 9.7071 & 33.6583 & 9.7071 \\
$K_{3}^{*}$ & 60 & 0.2 & \pm 65.7019 & 193.8010 & 4.8357 & 42.5678 & 4.8357 \\
\hline
\end{tabular}

\section{Experimental Results}

In order to illustrate how the proposed controller works, we consider the experimental setup consisting in two Phantom Omni haptic devices as the one depicted in Fig.6 (in a master/slave configuration), implemented by means of the Matlab-Simulink toolkit Phansim. It is also worth mentioning that the angular positions were normalized with respect to the mechanical stops of each joint. Now, using the stability analysis described above, we chose the controller's gains as $K=[20,2]^{T}, K=[20,1]^{T}$ and $K=[10,2]^{T}$ for the joint one, two and three, respectively. Furthermore, we propose an experimental test perceiving a plastic sphere as it is shown in figure 6 . This consists in manipulating the master device in order to "feel" the plastic sphere in a remote environment, where the slave device is located (it is worth to mention, that it is not necessary that the master and slave are located "close" to each-other. However, for illustrative purposes only we have chosen the proposed physical configuration for the visualization of the experiment). The experimental results are illustrated in figures 7(a), 7(b) and 8. Furthermore, Fig. 8 shows how the control scheme implemented drives the trajectory of the master device which is also guided by the human operator, following the path created by the human operator but restricted by the plastic sphere located in the remote environment, this creates the "feel" sensed by the human operator.

\section{COnCluding Remarks}

The bilateral control scheme using the $\mathrm{P}-\delta$ controller presented in this paper shows a good performance in terms of enabling a kinematic correspondence between the master and slave device. Furthermore, the closed-loop stability analysis method can be applied in a straightforward way. Therefore, this method can be easily implemented for practical applications. Finally, we aim to have experimental results showing a performance comparative between PID (Proportional- 


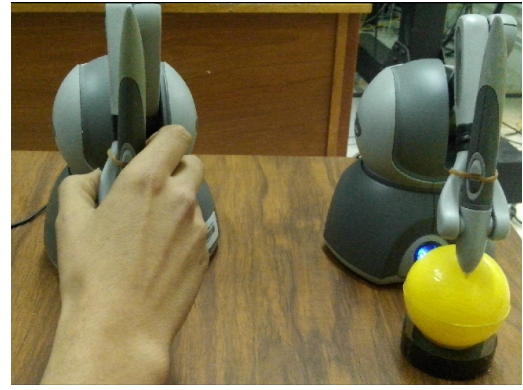

Fig. 6. Experimental setup.
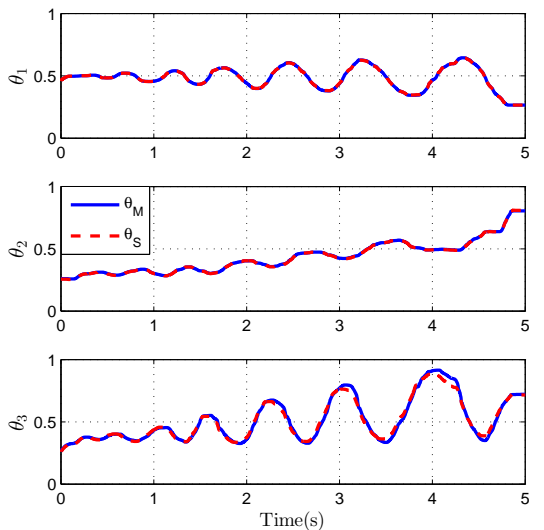

(a) Joints
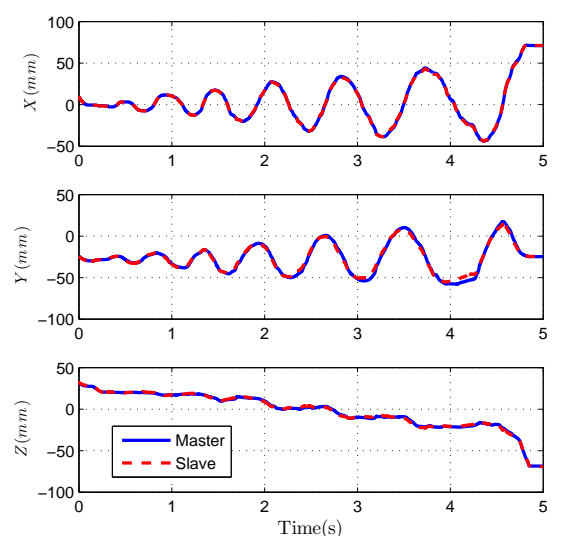

(b) Final Effector Position

Fig. 7. Total system response perceiving a sphere

Integral-Derivative) like controllers and the proposed $P-\delta$ controller for the final version of this paper.

\section{ACKNOWLEDGMENT}

This work is supported in part by DIGITEO-France and by the research grant PRODEP DSA/103.5/15/7488.

\section{REFERENCES}

[1] S. Skaar and C. F. Ruoff, "Teleoperation and robotics in space," in American Institute of Aeronautics and Astronautics, 1994.

[2] K. Kim, J. Park, H. Lee, and S. K., "Teleoperated cleaning robots for use in a highly radioactive environment of the dfdf," in Proc. SICEICASE International Joint Conference, 2006.

[3] M. Tavakoli, R. V. Patel, M. Moallem, and A. Aziminejad, Haptics for Teleoperated Surgical Robotic Systems. World Scientific Publishing Company, 2003.

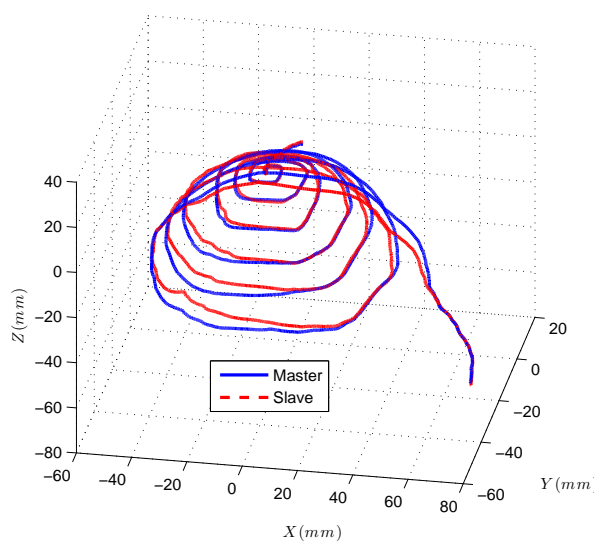

Fig. 8. Trajectory of the system under the perception of a sphere

[4] N. Tanner and G. Niemeyer, "Improving perception in time delayed telerobotics," International Journal of Robotics Research, 2005.

[5] L. Jones and I. Hunter, "Human operator perception of mechanical variables and their effects on tracking performance," ASME Advances in Robotics, 1992.

[6] K. Hashtrudi-Zaad and S. E. Salcudean, "Transparency in time-delayed systems and the effect of local force feedback for transparent teleoperation," IEEE Transactions on Robotics ans Automation, vol. 18, no. 1, pp. 31-38, 2002.

[7] S.-I. Niculescu and W. Michiels, "Stabilizing a chain of integrators using multiple delays," IEEE Trans. Aut. Control, vol. 49, no. 5, pp. 802-807, 2004.

[8] C.-I. Morărescu and S.-I. Niculescu, "Stability crossing curves of siso systems controlled by delayed output feedback," Dynamics of Continuous, Discrete and Impulsive Systems, 2007.

[9] B. Liacu, A. T. Koru, H. Ozbay, S.-I. Niculescu, and C. Andriot, "Optimizing low-order controllers for haptic systems under delayed feedback," Control Engineering Practice, vol. 21, pp. 655-668, 2013.

[10] J. Neimark, "D-subdivisions and spaces of quasi-polynomials," Prikl. Math. Mech., vol. 10, pp. 349-380, 1949

[11] S. Bhattacharyya, C. H., and L. Keel, Robust Control: The Parametric Approach. Prentice-Hall Information \& System Science Series, 1995.

[12] H. Guggenheimer, Differential Geometry. New York: Dover Publications, 1977

[13] W. Michiels and S. Niculescu, "Stability and stabilization of timedelay systems," in Advances in Design and Control, Philadelphia: SIAM, 1997.

[14] A. Mohammadi, M. Tavakoli, and A. Jazayeri, "Phansim: A simulink toolkit for the sensable phantom haptic device," in Proceedings of the 23rd CANCAM, 2008.

[15] S. Hirche and M. Buss, "Human perceived transparency with time delay," in Advances in Telerobotics, ser. Springer Tracts in Advanced Robotics, M. Ferre, M. Buss, R. Aracil, C. Melchiorri, and C. Balaguer, Eds. Springer Berlin Heidelberg, 2007, vol. 31, pp. 191-209.

[16] R. Datko, "A procedure for determination of the exponential stability of certain differential-difference equations," Quarterly of Applied Mathematics, 1978. 